\title{
LA ENFERMEDAD DEL ADYUVANTE EN RATAS INFECTADAS EXPERIMENTALMENTE CON TRYPANOSOMA CRUZI (*)
}

\author{
SHvia REVGh.li (1), Osear BotTASso (1), Gilda MORENo (2), José valenti (3), Analia \\ NOCTTO (3), Nelly AMERIO (1) \& Julio MORINI (1)
}

\section{R E S U M E N}

Se estudió la evolución de la artritis por adyuvante en ratas que habían sido infectadas previamente con Trypanosoma cruzi, con el objeto de evaluar su competencia inmunológica a través de la respuesta artrítica. La artritis por adyuvante se indujo en ratas adultas, endocriadas de ambos sexos, con $0.1 \mathrm{ml}$ de adyuvante completo de Freund en la almohadilla plantar, en 2 lotes: a) inyectadas 90 días antes con $1 \times 10^{6}$ T. cruzi y b) testigos normales simultáneos. Se midieron, la lesión artrítica macroscópicamente con una escala semicuantitativa, y con microscopía óptica la histopatología de la lesión local y la del corazón, a los 180 días post-infecuión. La magnitud de las lesiones artríticas en las ratas con T. cruzi fue significativamente menor $(p<0.001)$ que la de los testigos, en todo el periodo. El infiltrado inflamatorio local, formado por linfocitos, plasmocitos y macrófagos fue significativamente menor $(p<0.001)$ en las ratas chagásicas, con respecto al de los testigos. Se postula que en las ratas que recibieron $\mathbf{T}$. cruzi la respuesta artrítica menor podría deberse a una competición antigénica con los determinantes del parásito o a mecanismos inmunosupresores que interfieren en la producción de la entidad experimental.

UNITERMOS: Trypanosoma cruzi - Infecção experimental em ratos Adyuvante de Freund

\section{N T R O D U C C I O N}

En 1956 PEARSON ${ }^{6}$ demonstró que la inyec. ción intradérmica de adyúvante completo de Freund (ACF) en la pata producía artritis y periartritis en ratas, fenómeno que denominó artritis por adyuvante (AA). Posteriormente se comprobó que se trataba de una enfermedad generalizada, ya que en alguns casos estaba acompañada de iridociclitis, lesiones nodulares en oreja y cola, rash cutáneo, lesiones genitourinarias y diarrea ${ }^{7}$. WAKSMAN \& col. ${ }^{21}$ postularon que este fenómeno podría deberse a una reacción de hipersensibilidad retardada hacia la bacteria presente en el ACF o bien a algunos de sus constituyentes o sus metabolitos. En apoyo a esta teoría puede señalarse que la enfermedad se pudo transferir pasivamente con células de ganglio linfático, pero no con suero de animales sensibilizados. Además, se demostró que la misma puede inhibirse mediante la irradiación total $(600 \mathrm{r})$ del animal de expe rimentación o con el empleo de corticoides antes de la inyección del adyuvante. También

(*) Trabajo realizado en la División Inmunologia, Facultad de Clencias Médicas, Universidad Nacional de Rosarto Santa Fé 3100, Rosario (2000), Argentina

(1) Miembro de la Carrera del Investigador, UNR

(2) Miembro de la Carrera del Personal de Apoyo, CONICET

(3) Cátedra de Patologia, Facultad de Ciencias Médicas, vNR 
REVELLI, S.; BOTASSO, O.; MORENO, H.; VAIENTI, J.; NOCITO, A.; AMERIO, N. \& MORINI, J. - L L enfermedad del adyuvante en ratas infectadas experimentalmente con Trypanosoma cruzt. Rev. Inst. Med. trop. São Paulo, 28: 154-159, 1986 .

se verificó que si los animales se ponian en contacto con el bacilo de Koch o con la fracción Wax $\mathrm{D}$ del mismo, en los primeros días de vida $o$ antes del desafío con ACF, se podía inducir un mecanismo semejante a la tolerancia inmunológica $8,9,21$.

En nuestro laboratorio se ha desarrollado en una línea de ratas altamente endocriada, un modelo experimental que reproduce algunas de las caracteristicas de la enfermedad de Chagas crónica 11 con una parasitemia en la fase aguda que se extingue aproximadamente a los 40 días, curva de anticuerpos especifica crónica y desde ios 6 meses de la infección, lesiones histopatológicas de corazón en el $70 \%$ de los casos, compatibles en cierta medida con las observadas en la miocarditis chagásica 11,20. En este modelo, se decidió inducir la $\mathrm{AA}$ en los animales luego del período agudo de la infección chagásica, con el propósito de estudiar si la infección con Trypanosoma cruzi modifica los mecanismos inmuno-competentes comprometidos en la producción de esta patología.

\section{MATERIAL Y METODOS}

Animales - Se trabajó con ratas endocriadas, hembras y machos, de línea " 1 ", de 21-25 días de edad. Esta cepa deriva de ratas de linea e," IIM (i) que se crían en nuestro Bioterio desde 1963 y tiene actualmente 45 generaciones de endocria. Los animales permanecieron en las mismas condiciones experimentales durante el tiempo de la experiencia $y$ fueron divididos en 2 grupos: I) Infectados con $\mathbf{T}$. cruzi a los que se les indujo AA; II) Testigos con AA únicamente.

Infección experimental - Se utilizó la cepa Tulahuén de Trypanosoma cruzi, que se mantiene por pasajes subcutáneos en ratones de 21 días de edad. Las ratas fueron infectadas con $1 \times 10^{6}$ tripomastigotes por via subcutánea y en todos los casos se controló la presencia de parásitos en sangre el día $8^{\circ}$ post-inóculo.

Inducción y evaluación de la $\mathbf{A A}$ - La enfermedad se indujo inoculando al dia 90 postinfección con $T$. cruzi, una sola dosis $(0.1 \mathrm{ml})$

(1) Supplement IV of the Int. Survey on the Supply. Quality and Use of Laboratory Anmals, Int. Committe on Lab. Animals, Carshalton, England, 1964 de una suspensión oleosa de Mycobacterium tuberculosis (Cepa H $37 \mathrm{Ra}, 5 \mathrm{mg} / \mathrm{ml}$ Inmunoquemia) en la almohadilla plantar de la pata trasera derecha. Diez días más tarde, comenzaron los signos de AA que fueron evaluados por observación directa y estudios histológicos. En la observación directa se usó la siguiente escala semicuantitativa: tumefacción de las articulaciones pequeñas (interfalángicas, metacarpo y metatarsofalángicas) 1 punto; tumefacción de las articulaciones grandes (carpo, tarso y muñeca) 2 puntos; nódulos en cola 1, 2 y 3 según la extensión; conjuntivitis 1 punto; rash cutáneo y alteraciones crónicas de la piel 1 punto por lesión. Cuando la tumefacción fue el doble de lo observado habitualmente, se duplico el puntaje. Los 2 grupos experimentales fueron controlados desde el día 10 hasta el 40 post-inyección de ACF, ya que a partir de esa fecha las lesiones tendieron a estabilizarse.

Anticuerpos humorales - Los anticuerpos anti-T. cruzi se titularon al día 90 post-infeccrón con $T$. cruzi y 20 días después de la inducción de la AA. Se usó la reacción de hemaglutinación indirecta y la técnica de microtitulación (Polychaco SAIC, Técnica de Auerbach y Yanovsky). Los resultados se expresan como el $\log _{2}$ de la inversa de la dilución.

Estudio histológico de la AA - Se realizó a los 180 días posteriores a la infección con T. cruzi. Los animales se sacrificaron en cámara de éter. Se analizaron las patas posteriores afectadas por la inyección de ACF. El material fue fijado con líquido de Bouin, descalcificado con ácido nítrico al $10 \%$, se incluyó en parafina y se practicaron cortes de $6 \mu$ que fueron coloreados con hematoxilinaeosina y tricrónica de Gomori. La lesión fue evaluada con microscopía óptica en base a la distribución y extensión del infilltrado inflamatorio. Se clasificó en leve, moderada e intensa. Se consideró leve, a la presencia de un infiltrado de linfocitos, macrófagos y plasmocitos dispersos en la dermis e hipodermis; moderado, cuando el infiltrado confluente llegaba en profundidad hasta la fascia muscular superficial con presencia de granulomas de células epitelioides y células gigantes multinucleadas; intenso, cuando habia infiltrado denso que invadia el plano muscular estriado y llegaba a la sino 
REVELLI, S.; BOTASSO, O.; MORENO, H.; VALENTI, J.; NOCITO, A.; AMERIO, N. \& MORINI, J. - La enfermedad del adyuvante en ratas infectadas experimentalmente con Trypanosoma cruzi. Rev. Ynst. Med. trop. São Paulo, 28: 154-159, 1986.

vial y al plano óseo, con presencia de granulomas.

Histopatología de miocardio - En la autopsia se extrajo el corazón y se incluyó en parafina realizándose cortes transversales de $5 \mu$ de espesor que se colorearon con hematoxilina. eosina. En la histopatología cardíaca se estudiaron: 1) Miocarditis crónica focal (MCF), determinada por la presencia de acúmulos localizados de linfocitos con destrucción de las fibras miocárdicas. Según el grado de desarrollo de la lesión se clasificó en: a) leve: pequeño acúmulo de linfocitos con destrucción de 2-3 fibras miocárdicas; b) moderada: grupos de linfocitos con destrucción de pequeños grupos de fibras miocárdicas; c) intensa: acúmulos mayores de histiocitos y linfocitos con destrucción de grupos grandes de fibras miocárdicas. 2) Pericardicis (Pc), determinada por la presencia de un infiltrado inflamatorio crónico en el epicardio, según el grado de desarrollo se la clasificó en a) leve: pequeños grupos de linfocitos e histiocitos dispersos; b) moderada: cuando el infiltrado formaba una masa nodular pequeña; c) intensa: cuando el infiltrado formaba una placa extensa. 3) Fibrosis (Fi), determinada por la presencia de bandas de tejido conectivo en reemplazo de las fibras miocárdicas. Segun el grado de desarrollo y la extensión en el miocardio se clasificó en leve, moderada o intensa. La presencia de MCF, Pc ó Fi se agrupó como lesión cardíaca (Lc).

Anãlisis estadístico - Los resultados obtenidos en la evaluación macroscópica se analizaron con la prueba U de Mann-Whitney y los correspondientes a la evaluación microscópica por la prueba $G$. Los títulos de Ac anti-Tc se estudiaron con la " $t$ " de Student para datos apareados 15,16 .

\section{RESULTADOS}

Evolución de la AA - Los primeros signos artríticos comenzaron a aparecer entre el $70^{\circ} \mathrm{y}$ 10. día posterior al inóculo del ACF, especialmente en las patas posteriores y de ellas con mayor intensidad en la inoculada con el ACF. Es necesario aclarar que en la línea de ratas utilizadas para este experimento la AA se visualiza principalmente con signos artríticos. Las manifestaciones extraarticulares tales como conjuntivitis, rash cutáneo y lesiones costrosas de la piel se observaron con más frecuencia en los animales testigos

En la Fig. 1 se puede observar la evolución de la $\mathrm{AA}$ en los 2 grupos experimentales. Se muestra que los animales inoculados con Tc desarrollaron una patología significativamente menor ( $p<0.001$ ) que los testigos, en todos los días estudiados.

Además, se puede ver que en los animales previamente infectados en los cuales se indujo la AA, desaparece la diferencia en el comportamiento entre macho y hembras, fenómeno que es evidente en los testigos ( $\mathrm{Ej}$. dia 23 post-inducción AA, $\mathrm{p}<0.02$ ).

A partir del día 30 post-inóculo del ACF las lesiones tienden a regresar lentamente y al día 90 del proceso artrítico, cuando los animales fueron sacrificados para el estudio histológico, se mantenían aún las diferencias entre los grupos experimentales.

Anticuerpos humorales anti-T. cruzi - El título de anticuerpos de los animales infecta. dos con T. cruzi antes del inóculo con ACF fue de: $\bar{x} \pm$ e.s. $=5.0 \pm 0.29 ; n=15 ; 20$ días después del adyuvante, aumentó significativamente: $\bar{x} \pm$ e.s. $=6.64 \pm 0.23 ; n=14(p<$ 0.001 ), En los testigos, si bien los títulos de Ac son inespecíficos: $\bar{x} \pm$ e.s. $\pm 2.12 \pm 0.20$; $\mathrm{n}=16$, la inducción de la AA produjo un leve ascenso: $\vec{x} \pm$ e.s. $=3.0 \pm 0.25 ; n=13(p<$ $0.05)$. Estos incrementos podrían deberse a un efecto inespecífico del ACF.

Hisiopatología - En el lugar del inóculo del $A C F$ se encontró un infiltrado celuuar denso, compuesto por linfocitos, plasmocitos y macró fagos. Se observó con mucha frecuencia acúmulos de células epitelioides y células gigantes multinucleadas tipo Langhans, configurando granulomas sin caseosis. In algunos casos se constató arteritis en vasos pequeños con elementos linfoides en plena pared vascular. La cuantificación de las lesiones figura en la Tabla I. Se puede verificar que las ratas infectadas con T. cruzi desarrollaron un infiltrado infiltrado inflamatorio significativamente menor que el de los testigos $(p<0.001)$, en los que en todos los casos fue de tipo III. 
REVELLI, S.; BOTASSO, O.; MORENO, H.; VALENTI, J.; NOCITO, A.; AMERIO, N. \& MORINI, J. - La enfermedad del adyuvante en ratas infectadas experimentalmente con Trypanosoma crud. Rev. Inst. Med. trop. Säo Paulo, 28: 154-159, 1986.
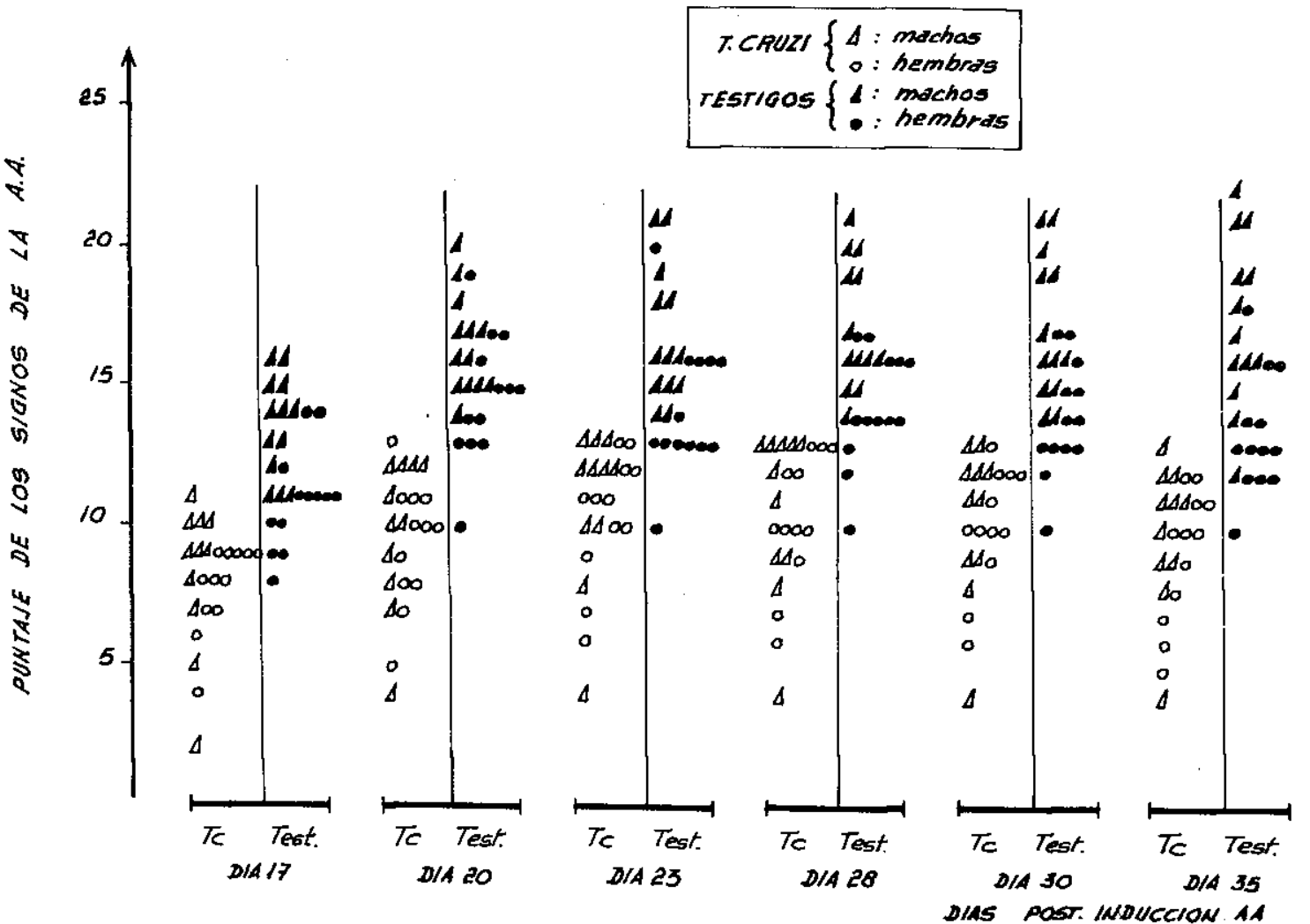

Fig. 1 - Evolución de la artritis por adyuvante (AA) en ratas infectadas con T. cruzd y testigos, mostrando los datos indi viduales. La diferencia en la magnitude de la lesion entre ambos grupos es altamente significativa en todo el perfodo (p $<0.001$ ).

T A $\mathbf{B}$ 工

Porcentaje de lesión artrítica en ratas chagásicas $y$ testigos (Evaluación Microscópica)

\begin{tabular}{lcccc}
\hline Grupos Experimentales & \multicolumn{4}{c}{ Grado do lesión } \\
& I & II & III & P \\
\hline & 0 & 0 & 100 \\
Testlgos de $A A(*)$ & $(0 / 21)$ & $(0 / 21)$ & $(21 / 21)$ & $<0.001$ \\
\hline & 47.4 & 42.1 & 10.5 \\
Chagásicos $+A A(*)$ & $(9 / 19)$ & $(8 / 19)$ & $(2 / 19)$ \\
\hline
\end{tabular}

(*) Artritis por adyuvante, inducids en la pats trasera con A.C.F.

(*) Inoculadas 90 dias antes de la inducción con $T$. cruwl Grado de lesión: I, leve; II, moderada; III, intensa. Ver descripción en el texto.

El estudio histopatológico del miocardio mostró que sólo el $25 \%$ de los animales infectados con $\mathrm{Tc}$ y a los que se les indujo $\mathrm{AA}$, de sarrollaron una miocarơitis crónica focal, porcentaje que es muy inferior al que se obtiene en el modelo crónico 11,20 . Las ratas con AA so. lamente, presentaron un $8 \%$ de miocarditis, similar al de los testigos sin ningún tratamiento.

\section{DISCUSIÓN}

De acuerdo con nuestros resultados, en esta línea de ratas, la lesión es predominantemente granulomatosa crónica, lo cual constituye una típica expresión de la inmunidad mediada por células (IMC) 22 . Como ya fuera enunciado, WAKSMAN \& col. 21 postularon que la AA se produce por un mecanismo de hipersensibilidad retardada. TAUROG \& $\mathrm{col}^{17}$ señalaron que la artritis por el ACF está mediada por una subclase de linfocitos $T$ productores do linfoquinas. Mas aun KAYASHIMA \& col.4 postulan que participan 2 subpoblaciones de linfocitos $T$ en el desarrollo de la AA, una encargada de producirla (sensible al suero anti-timo cito) y otra de linfocitos $T$ de corta vida que ejerce efecto supresor en la inducción. PEAR. 
REVIMLI, S.; BOTASSO, O.; MORENO, H.; VALENTI, J.; NOCITO, A.; AMERIO, N. \& MORINI, J. - La enfermedad del adyuvante on ratas infectades experimentalmente con Trypanosoma cruzi. Rev. Inst. Med. trop. São Paulo, 28: 154-159, 1986.

SON \& $\mathrm{col} .^{9}$ señalaron que esta entidad podria ser um fenómeno autoinmune, to cual refuerza lo anterior, puesto que la IMC es primordial para la producción de enfermedades autoalergicas experimentales 22 .

En este experimento, se ha detectado que los machos desarrollan un proceso mas intenso que las hembras, fenomeno que no se constata en los animales infectados con $\mathbf{T}$. cruzi, probablemente debido a la escasa magnitud de la lesión. Estas diferencias hacen suponer que la capacidad para desarrollar el proceso artritico seria un caracter influido por el sexo.

Los resultados expuestos evidencian que las ratas infectadas con $\mathbf{T}$. cruzi desarrolian un proceso de menor intensidad en relación a los testigos, que lleva a postular la existencia de mecanismos posibles capaces de interferir con la respuesta inmune.

En primer têrmino, podria tratarse de una competición antigénica secuencial ${ }^{18}$, donde los antígenos parasitarios (dominantes) actuarían inhibiendo la respuesta hacia constituyentes antigénicos de la micobacteria (suprimidos). Este proceso de competición entre antígenos, eventualmente, podria ser operativo en otro nivel. Así, WAKSMAN \& col. ${ }^{21}$ han observado que al tratar de inducir en un mismo animal la AA y la encefalomielitis alérgica experimental (EAE), la artritis fue mas frecuente en las ratas que no desarrollaban la EAE, postulándose una competición de antigenos en la sensibilización. Si tenemos en cuenta que estudios en ratones infectados con $\mathbf{T}$. cruzi sugieren la participación de un fenomeno de autoinmunidad en la producción del daño tisular miocárdico ${ }^{2}$, 5,19 , en nuestro caso, podría suponerse también la presencia de un mecanismo de competición entre los antigenos inductores de la miocarditis y la artritis (similar a lo observado entre la AA y EAE), que podría explicar la baja incidencia de la lesión cardiaca en los animales con ambas patologias.

El segundo aspecto a considerar en la físio. patologia de la infección experimental con $\mathbf{r}$. cruzi es el de la inmunosupresión. Diversos .1utores han detectado la supresión tanto humoral como celular de la respuesta inmune especifica e inespecifica en la fase aguda de la infección murina $3,10,12,13$, y recientemente SCOTT ${ }^{14}$ ha observado una supresión especifica de la IMC en un modelo crónico. Resultados preliminares đemouestran que la menor respusta artritogénica puede transferirse a un receptor $\sin$. geneico normal con células de bazo de rata infectada con T. cruzi (datos no publicados). Asimismo, hemos comprobado que la respuesta de hipersensibilidad tardia hacia globulos rojos de carnero, se halla deprimida en esta misma linea de ratas que cursan la infección ttripanosomiasica ${ }^{1}$. Por lo tanto cabría la posibilidad de que se produzcan mecanismos inmunosupresores que interfieran en la competencia inmunológica necesaria para producir esta entidad experimental.

\section{SUMMARY}

\section{The adjuvant disease in rats experimentally infected with Trypanosoma cruzi}

The experimental arthritis produced in rats which had been infected at weaning with Try. panosoma cruzi was studied 90 days after inoculation. Focal arthritis was induced by injecting $0.1 \mathrm{ml}$ of Freund complete adjuvant in one hind foot-pac of inbred previously treated animals and controls. To measure different degrees of foot-pad inflammation and swelling a semi quantitative scale was used. Furthermore, a histopathological study of heart and joints was performed 180 days after $T$. cruxi inocula. tion. The joint injury in the T. cruxi group was significantly smaller than in the controls, during the acute period ( $\mathrm{p}<0.001$ ).

Histopathologic observations showed local tissue inflammatory infiltration with lymphocytes, plasma cells and macrophages. These pathological changes observed in treated animals were also smaller than in the controls ( $p<$ 0.001 ). It is postulated that in chagasic rats the less severe arthritis could be due to an antigenic competition or immunosuppressive mechanisms present in infected animals.

\section{AGRADEGIMIENTOS}

Trabajo apoyado con subsidio de SECYT, Argentina y Consejo de Investigaciones de la UNR. Los Autores agradecen a la estadistica C. 
REVELLI. S.; BOTASSO, O.: MORENO, H.; VALENTI, J.; NOCITO, A.; AMERIO, N. \& MORINI, J, - La enfermedad del adyuvante en ratas infectadas experimentalmente con Trypanosoma cruzil. Rev. Inst. Med. trop. São Paulo, 28: 154-159, 1986.

Tarrés su asesoramiento, al Sr. H. Poli y Srta. B. Scelfo su esmerada colaboracíón técnica y a Lab. Polychaco por la provisión de reactivos.

\section{REFERENGIAS}

1. BOTTASSO, O. A.; REVELLI, S.; MORENO, H.; SCELLO, B.; POLI, H. \& MORINI, J. C. - Respuesta inmuno a glóbulos rojos de carnero (GRC) en ratas infectadas con Trypanosoma cruvi (TC). Actas XXIX Reunión Cientifica de la SAIC, 1984.

2. COSSIO, P. M.; DIEZ, C.; LAGUNES, R. P. \& ARANA, R. M. - Inmunopatologia de la Enfermedad de Cha. gas. Hechos y perspectivas. Medicina (B. Alres), 40: 222-230, 1980.

3. CUNNINNGHAM, D. S.; KUHN, R. E. \& ROWLAND, E. C. - Suppression of humoral responses during Trypanosoma cruxi infections in mice. Infect. Immun., 22: 155-160, 1978.

4. KAYASHIMA, K.; KOGA, T. \& ONOUE, K. - Role of $T$ lymphocytes in adfuvant Arthritis. II. Different subpopulations of $\mathbf{T}$ lymphocytes functioning in the development of the disease. J. Immunol., 120: 1127-1131, 1978.

5. LaGUeNS, R. P.; CABEZA MRCKERT, P.; ChaMBo, G. \& GELPI, R. J. - Chronic Chagas disease in the mouse. II. Transfer of the heart disease by means of immunocompotent cells. Medicina (B Aires), 41: 4043, 1881.

6. PEARSON, C. M. - Development of arthritis, periarthritis, and periostitis in rats given adjuvants. Proc. Soc. exp. Biol. (N.Y.), 91: 95-101, 1956.

7. PEARSON, C. M.; WaKSMan, B. H. \& SHARP, J. T. - Studies of arthritis and other lesions induced in rats by injection of mycobacterial adjuvant. $v$. Changes affecting the skin and mucous membranes. Comparison of the experimental process with human disease. J. exp. Med., 113: 485-509, 1961.

8. PEARSON, C. M. \& WOOD, F. D. - Factors wich modify adjuvant-induced arthritis. Arthr. and Rheum.. 5: $654,1962$.

9. Pearson, C. M. \& WoOD, F. D. - Passive transfer of adjuvant arthritis by lymph node cells. J. exp. Med., 120: 547-560, 1964.

10. RAMOS, C.; LAMOYI, E.; FEOLI, M.; RODRIGUEZ, M.; PEREZ, M. \& ORTIZ-ORTIZ, L. - Trypanosoma cruzi: immunosuppressed response to different antigens in the infected mouso. Exp. Paraglt., 45: 190-199, 1978.
11. REVELLI, S. S.; AMBRIO, N.; MORENO, H. S.; VA. LENTI, J. L.; BALBARREY, H. \& MORINI, J. C. Enfermedad de Chagas crónica en la rata. Caracteristicas serológicas, electrocardiográficas e histopatológcas. Medicina (B. Aires), 40: 69.78, 1980.

12. REED, S. G.; LARSON, C. L. \& SPEER, C. A. Contact sensitivity responses in mice infected with Trypanosoma cruzi. Infect. Xmmun., 22: 548-554, 1978.

13. ROWLAND, E. C. \& KUHN, R. E. - Suppression of cellular responses in mice during Tryponosoma cravd infections. Infect. Immun., 22: 393-397, 1978.

14. SCOTT, M. T. - Delayed hypersensitivity to Tryplnosoma cruvf in mice: specific suppressor cells in chronic infections. Immunology, 44: 409-417, 1981.

15. SIEGEL, S. - Estadística no paramétrica. 2da. Edición española. México, Edit. Trillas, 1979. p. 143-155.

16. SOKAI, R. R. \& ROHIF, F. J. - Biometría. Madrid, H. Blume Ediciones, 1979. p. 186-192, 611-613.

17. TAUROG, J. D.; SANDBERG, G. P. \& MAHOWALD, M. L. - The cellular basis on adjuvant Arthritis. II. Characterization of the cells mediating passive trans. fer. Cell. Immunol., 80: 198.204, 1983.

18. TAUSSIG, M. J. - Antigenic competition. In: SELA, M., ed. - The antigens. New York, Academic Press, 1977. v. 4 , p. 333-368.

19. TEIXEIRA, A. R. L. - Chegas' diserse: trends in im. munological research and prospects for immunoprophy. laxis. Bull. Wld. Hith. Org., 57: 697-710, 1979.

20. VALENTI, J. L.;; REVETII, S. S.; MORINT, J. C. \& BURGOS, M. - Evolución de las slteraciones cardíacas en un modelo de Enfermedad de Chagas crónica en la rata. Mediclna (B. Aires), 40: 810, 1980.

21. WAKSMaN, B. H.; PEARSON, C. M. \& SHARP, J. T. - Studies of arthritis and other lesions induced in rats by injection of mycobacterial adjuvant. II. Evidence that the disease is $\mathbf{a}$ disseminated immunologic response to exogenous antigen. J. Immunol., 8 s: 403-417, 1960 .

22. WAKSMAN, B. H. - Cellular hypersensitivity and itnmunity: conceptual changes in last decade. Cell. Immunol., 42: 155-169. 1979.

Recebido pars publicaçäo en 29/5/1985. 